\title{
Application and analysis of image modeling based on UAV
}

\author{
Shuheng Wang ${ }^{1}$, and Xuefeng $\mathrm{Niu}^{1, *}$, and Chunyu $\mathrm{Zhu}^{2}$, and Xiang $\mathrm{Wu}^{3}$, and Shi $\mathrm{Liu}^{4}$ \\ ${ }^{1}$ College of Geo-Exploration Science and Technology, Jilin University, Changchun, 130026, China \\ ${ }^{2}$ Jilin Institute of Geo-information, Changchun, 130062, China
}

\begin{abstract}
As one of the important geographic information products, orthophotos play an extremely important role in the field of geographic information. With the rapid development of China's economic construction and the continuous improvement of photogrammetry technology, traditional orthophoto has been replaced by real orthophoto to meet the requirements of large-scale accurate mapping. This article discusses the method of generating real shot images, uses drone images, and uses Zhanwei New Village in Wuhan as the survey area to lay out the ground control points. Based on Pix4D modeling, the air and three encryptions are completed and based on high-precision DSM Quickly generate real radiography products. The research in this paper shows that the corrected real image can eliminate the phenomenon of blocking the wall and the problem of occlusion. It has a good effect and can be used in the field of line drawing maps. It provides a simple and quick solution for the rapid acquisition of orthophotos in the field of photogrammetry.
\end{abstract}

\section{Introduction}

With the rapid development of digital cities and intelligent surveying and mapping, the application of photogrammetry technology in geographic information has been continuously improved. Orthophoto is one of the most basic geographic information products, which is widely used in large-scale surveying and mapping, marine monitoring, land dynamic monitoring, as well as areas such as topographic map updates, all have played a very important role. At present, the production of orthophotos has attracted extensive attention from scholars at home and abroad. Li Deren [1] et al, proposed related theories of the DOM database. Through projective processing of the data, the acquisition of three-dimensional information on the terrain surface and no object Stereoscopic observation. Based on the corrected digital raster map, Zhang Jixian et al [2] studied the production method of remote sensing orthophotos without DEM support, and widely applied it to the construction of basic geographic information in China and related production. In order to solve the problem of TDOM production in large-scale cities, Deng Fei and Zhang Jianqing [3-4], etc., successively proposed a DBM-based overall projection and triangulation-based method for shadow detection,provides a convenient solution for quickly obtaining the oblique projection and shadowing problems of buildings, Ma Dongling [5,19], etc., based on the relevant production practices, studied the principles and methods of making orthophotos based on Inpho software, and concluded that Inpho series software can appropriately simplify the operation method and improve orthophoto Image production accuracy and operating efficiency. Zhang Ping [6] and others took the DOM production of Huai'an measurement area of 1: 2000 as an example in Fuzhou as an example, discussed the image mosaic and DOM production principles, and summarized the production technology and related principles. Yang Jingyu, Zhang Xuewen [7-8] et al, conducted research on the production of orthophotos, and proposed Z-PASS and the improved Z-BUFFER algorithm to accurately determine the masked area of orthophotos and completed the monitoring of DSM The visibility of grid points improves the accuracy of orthophoto production. Zhou Xiaomin [9], referring to the related problems of tilt photogrammetry in recent years, put forward the method of using StreetFactory system combined with aerial triangulation to achieve integrated regional network adjustment, and finally complete the establishment of high-precision 3D model. Fan Binbin [10] and others took Wuxiake Tielek Village in Luntai County as the research area and completed orthorectification based on DSM. The accuracy of the generated true radiographic image was higher than the relevant requirements of 1: 1000, and greatly Improve the efficiency of cartography. Feng Xiuli [11] et al, talked about using SPOT satellite data as an example, and with the support of ERDAS software, discussed how to use satellite data to make TDOM, and can be widely used in land resource management, urban and rural planning and other fields. With the support of PCI Geomatica software, Xia Tao [12] and others realized the automatic extraction of DEM and DOM information from ASTER data, and compared with the 1: 50000 measured map, and studied the accuracy of orthophoto. Liu Xiaoming [13] completed the correction of orthophotos to the ground by studying the characteristics of digital image maps and the process of orthophoto production, and discussed the development trend after orthophotos. Lucieer and AI M Y [17-18] used multi-view stereo vision algorithms and motion recovery structure algorithms to establish ultra-high resolution 3D

$\overline{{ }^{*} \text { Corresponding author:niuxf@jlu.edu.cn }}$ 
models. The generated orthophotos were mainly used to evaluate the survival status of Antarctic moss.

The above scholars mainly studied the related software required for real shooting images and the production principle of real shooting images, and explained in detail the related accuracy and existing problems of making real shooting. In this experiment, Zhanwei Xincun in Wuhan was used as the research area. UAV aerial photography was used to collect data, set up image control points, complete the air and space encryption based on PIX4D modeling and generate point clouds, and generate and edit digital surface models. On the basis of DSM, it can quickly generate related products of real orthophotos, and verify and analyze the results of the generated orthophotos, which solves the problems of tilting and partial occlusion of the house, thus providing a simple way to quickly obtain orthophotos in the field of photogrammetry Shortcut.

\section{Overview of the study area and data preparation}

Before making orthophoto maps, the following preparations need to be made [15-16]: (1) survey area overview and image data of the study area; (2) camera parameters and drone model: the camera parameters mainly include the height and width of the image, and Pixel size, etc; (3) Ground control point layout and aerial photo quality: The control point layout is realized by collecting and investigating topographic maps and control point results, and the aerial photo quality should strictly control the heading and lateral overlap to facilitate subsequent orthophotos Production. Among them, the layout of control points and the quality of aerial photos are the focus of this data preparation.

\subsection{Overview of the study area}

The research area selected in this experiment is Zhanwei New Village in Hongshan District, Wuhan City, Hubei Province. The survey area is located in the southeast of Wuhan City. Most of them are residential buildings and roads. The terrain is relatively flat, suitable for testing the problem of housing shelter; The flight path is a serpentine flight, the area of the study area is about $0.323 \mathrm{~km} 2$, the weather on the day of shooting is clear, The aerial photography of the aircraft was breezeless, cloudless, and the weather was clear. It is very suitable for using DJI UAVs to carry out aerial photography at different heights, different positions and angles to obtain building image data.

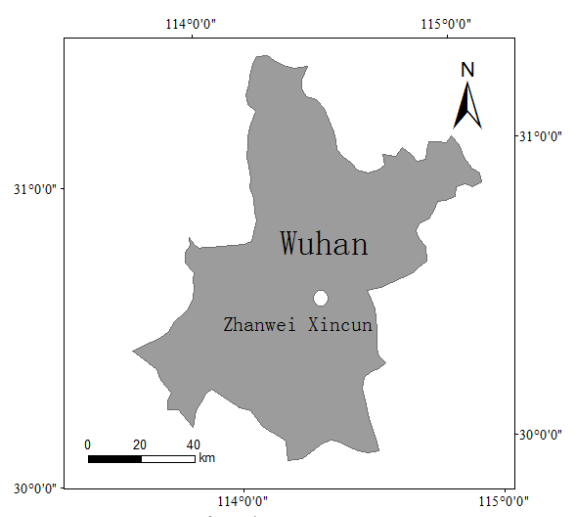

Fig. 1. Test area

\subsection{Data preparation}

\subsubsection{Camera parameters and drone model}

This test image was taken by DJI UAV. The model of the UAV is: DJI-FC6310R; the camera parameters are shown in Table 1:

Table 1. DJI-FC6310R camera parameters

\begin{tabular}{|l|l|l|l|}
\hline $\begin{array}{l}\text { Image width } \\
\text { (pixels) }\end{array}$ & 5472 & $\begin{array}{l}\text { Camera center } \\
\text { point Y (pixels) }\end{array}$ & 1831.48 \\
\hline $\begin{array}{l}\text { Image height } \\
\text { (pixels) }\end{array}$ & 3468 & $\begin{array}{l}\text { Camera sensor } \\
\text { width (mm) }\end{array}$ & 13.1328 \\
\hline $\begin{array}{l}\text { Camera focal } \\
\text { length (pixels) }\end{array}$ & $\begin{array}{l}3639 . \\
89\end{array}$ & $\begin{array}{l}\text { Camera sensor } \\
\text { height (mm) }\end{array}$ & 8.7552 \\
\hline $\begin{array}{l}\text { Camera center } \\
\text { point X (pixels) }\end{array}$ & $\begin{array}{l}2736 . \\
22\end{array}$ & Pixel size $(\boldsymbol{\mu m})$ & 2.4 \\
\hline
\end{tabular}

\subsubsection{Arrangement of control points and aerial quality}

The control points are laid out according to the actual conditions of the study area. Look for obvious object points in the survey area. The control points are evenly distributed along the route. The control points are located at the main trunk roads and house inflection points of Zhanwei Xincun and the intersection of roads. Observing their coordinates is conducive to air-to-air encryption. This drone flight has a total of 9 routes, flying in a serpentine shape. A total of 182 images are shot, and a total of 5 image control points are deployed to make real shooting images. The flight trajectory is shown below.

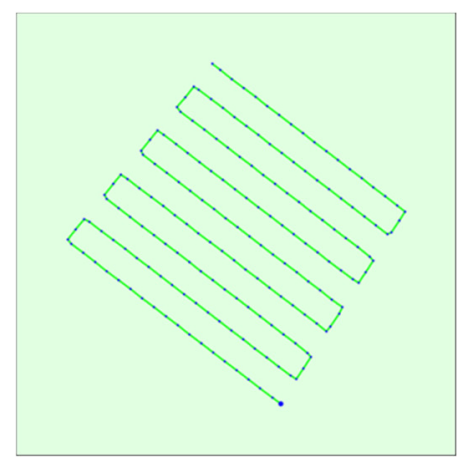

Fig. 2. The flight path of the uav 
The ideal degree of data acquisition directly determines the production effect of orthophotos. The flight requirements of traditional orthophoto aerial photography are different from those of real orthophotos. Among them, the data requirements for this aerial photo: the heading overlap must be at least $80 \%$, and the side overlap must be at least $60 \%$, and check whether there are breakpoints in the route and whether the images in the route are misaligned, and the base height ratio $(\mathrm{B} / \mathrm{H}) \mathrm{It}$ should be less than 0.3 , and ensure that there is a $3^{\circ}$ overlap in heading and sideways respectively. The requirements for the overlap of different types of cameras are as follows:

Table 2. Different camera overlap requirements

\begin{tabular}{|c|c|c|}
\hline Category & TDOM & $\begin{array}{c}\text { General } \\
\text { orthophoto }\end{array}$ \\
\hline ADS40 & $\begin{array}{c}\text { Lateral direction is } \\
\text { greater than } 70 \%\end{array}$ & $30-50 \%$ lateral \\
\hline DMC & $70-80 \%$ & $30-60 \%$ \\
\hline UCD & $68-75 \%$ & $30-60 \%$ \\
\hline $\begin{array}{c}\text { 150mm frame } \\
\text { type }\end{array}$ & $80 \%$ & $30-60 \%$ \\
\hline $\begin{array}{c}\text { 300mm frame } \\
\text { type }\end{array}$ & $80 \%$ & $30-60 \%$ \\
\hline
\end{tabular}

\section{Production of real shot images}

\subsection{Air three encryption and 3D point cloud generation}

Aerial triangulation is the main method of air-to-air encryption. The common method today is to deploy image control points with certain representative properties in the field. Among them, the coordinate system used in this data three encryption is the CGCS2000 coordinate system, and the POS data of the drone image is imported from the file, so that the position information can accurately match the image. This projection is a Gauss-Kruger $3^{\circ}$ band projection, and the central meridian is $114^{\circ}$ east longitude. The data is shown in Table 3: Air triangulation is the main method of encryption of the air and air. The common method today is to represent the field layout. The nature of the image control point, the industry's method of empty three encryption to generate six internal orientation elements of each photo. Among them, the coordinate system used in this data three encryption is the CGCS2000 coordinate system, and the POS data of the drone image is imported from the file, so that the position information and the image can be accurately matched. This projection is a Gauss-Kruger $3^{\circ}$ band projection, and the central meridian is $114^{\circ}$ east longitude. The data is shown in Table 3:

Table 3.Coordinate system and central meridian

\begin{tabular}{|c|c|}
\hline $\begin{array}{c}\text { Image } \\
\text { Coordinate System }\end{array}$ & WGS 84 \\
\hline $\begin{array}{c}\text { Output } \\
\text { Coordinate System }\end{array}$ & $\begin{array}{c}\text { CGCS2000/3-degree } \\
\text { Gauss-Kruger CM114E }\end{array}$ \\
\hline
\end{tabular}

This experiment uses Pix4D to complete automatic relative orientation and matching of image connection points. The modeling process is mainly divided into three parts [14]: first, input data first and edit related image attribute information; second, find relevant templates and parameters used for this experiment, one-click automatic empty three calculations For orientation elements, the point cloud matching is completed after the three-pass encryption test is passed, and a high-density three-dimensional point cloud is generated. Third, texture is added to the point cloud, and the texture mapping of the model is completed on the generated triangular mesh, and finally Related models after completion. Figure 3 is a schematic diagram of densely matching three-dimensional point clouds; Figure 4 is a schematic diagram of PIX4D-based empty three encryption.

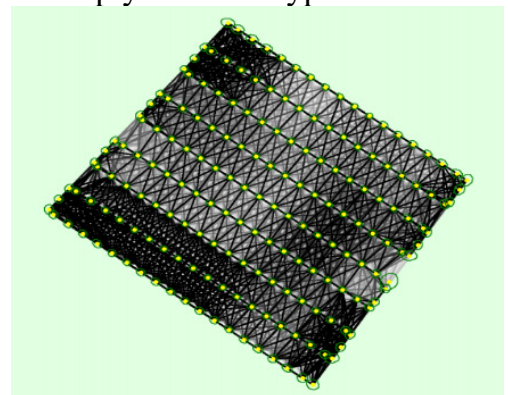

Fig. 3. Densely matched 3d point clouds

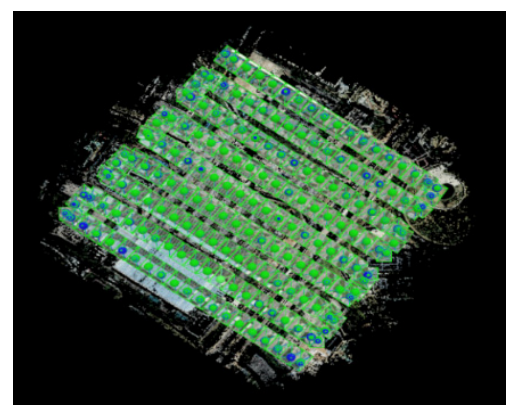

Fig. 4. Empty three encryption diagram

\subsection{DSM generation}

The photos with large overlapping degree obtained by using cross routes are used to generate the preliminary DSM through the multi-primitive and multi-image matching method. Secondly, the generated DSM is used to detect the occlusion area; then the occlusion area is analyzed and the texture is repaired to restore the stereo angle of the building [21]; Finally, the DSM editing is mainly done manually under the three-dimensional model to obtain high-precision DSM. Figures 5 and 6 show the actual terrain and the high-accuracy DSM editing process [22].

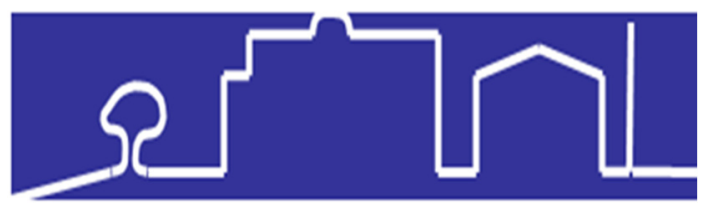

Fig. 5.The actual terrain 


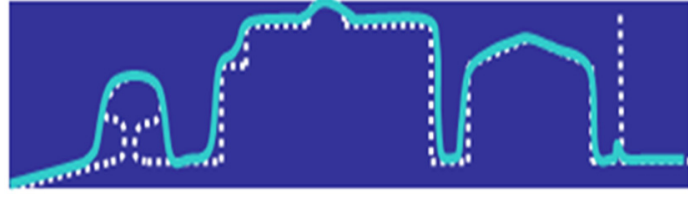

Fig. 6.Generated DSM profile

The biggest difference between making TDOM and traditional orthophotos is that TDOM orthorectification is based on DSM and DEM model. Therefore, the accuracy of the DSM and the production process directly affect the final result of the real shot image [20]. This article describes the generation process of DSM based on PIX4D three-dimensional modeling, and completes the corresponding occlusion analysis and texture repair. As shown in Figure 7.

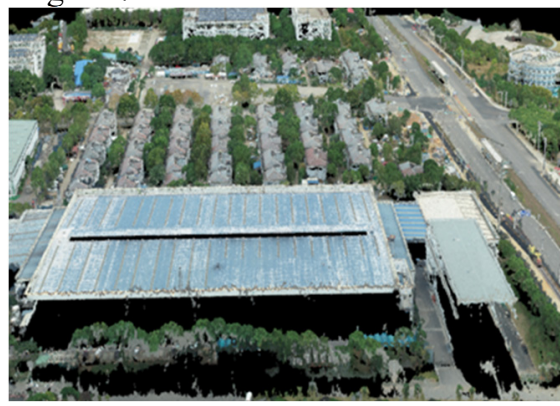

Fig. 7. Digital surface model

\subsection{Generation of real shot images}

This article discusses the method of making real shot images, and uses digital differential correction to eliminate all parallax. The resulting building maintains a vertical viewing angle and establishes a vertical view of the surface landscape, thereby effectively avoiding house tilting and shadowing. The impact of the district has restored the correct position of the building and completed the production of TDOM. The original image of this test is shown in Fig. 8, in which there is an obscured part and the house is tilted. In this paper, Pix4D three-dimensional modeling generates a point cloud to complete the three-space encryption, and on the basis of high-precision DSM, the corrected real shot image is shown in Figure 9, the wall tilt and the house shadowing phenomenon have been eliminated, and a vertical viewing angle has been established. The surface landscape, complete the production of real shooting images. It can be seen that the real radiographic image can be used in the field of line drawing maps, and the effect is better, which provides a simple and quick solution for the rapid acquisition of orthophoto images in the field of photogrammetry.

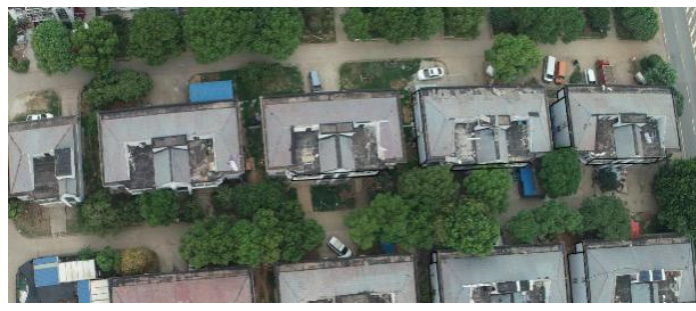

Fig. 8. The original image

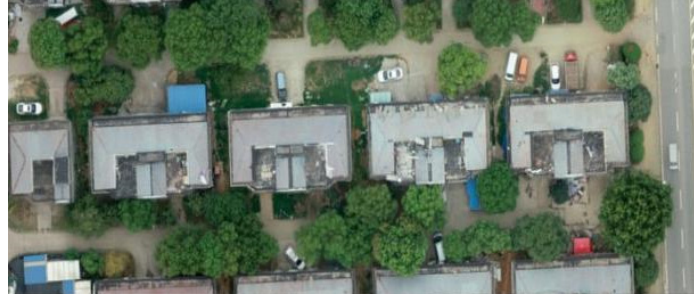

Fig. 9.Real projective image

\section{Accuracy evaluation of experimental $r$ esults}

For the model built, the accuracy evaluation is mainly carried out from the four aspects of regional cyberspace error, self-checking camera error, relative geographic variance and absolute geographic variance.

The third row shown in Table 4 is the error of air-to-air encryption, in pixels. The larger the pixel, the lower the accuracy, and the smaller the pixel, the higher the accuracy. , From this we can see that the accuracy is higher.

Table 4.Quality report form 1

\begin{tabular}{|c|c|}
\hline $\begin{array}{c}\text { Number of 2DKeypointObservations for } \\
\text { Bundle Block Adjustment }\end{array}$ & 3220420 \\
\hline $\begin{array}{c}\text { Number of 3DPoints for Bundle Block } \\
\text { Adjustment }\end{array}$ & 1035854 \\
\hline Mean Reprojection Error [pixels] & 0.175 \\
\hline
\end{tabular}

Table 5 shows the self-checking camera error. Due to the good initial camera parameter settings, the value difference between the upper and lower parameters of Initial Values and Optimized Values is not large. It can be seen from the figure that the three parameters R1, R2, R3 are not greater than 1 . Therefore, there is no distortion in the lens, and the camera parameters meet the requirements in this test.

Table 5. Quality report form 2

\begin{tabular}{|l|l|l|l|}
\hline & $\begin{array}{l}\text { Initial } \\
\text { Values }\end{array}$ & $\begin{array}{l}\text { Optimized } \\
\text { Values }\end{array}$ & $\begin{array}{l}\text { Uncertainties } \\
\text { (Sigma) }\end{array}$ \\
\hline $\begin{array}{l}\text { Focal } \\
\text { Length }\end{array}$ & $\begin{array}{l}3639.890 \\
\text { [pixel] } \\
8.736[\mathrm{~mm}]\end{array}$ & $\begin{array}{l}3649.968 \\
\text { [pixel] } \\
8.760[\mathrm{~mm}]\end{array}$ & $\begin{array}{l}2.890 \quad[\mathrm{pixel}] \\
0.007[\mathrm{~mm}]\end{array}$ \\
\hline $\begin{array}{l}\text { Principal } \\
\text { Point } \mathbf{x}\end{array}$ & $\begin{array}{l}2736.220 \\
{[\text { pixel] }} \\
6.567[\mathrm{~mm}]\end{array}$ & $\begin{array}{l}2750.661 \\
\text { [pixel] } \\
6.602[\mathrm{~mm}]\end{array}$ & $\begin{array}{l}0.064 \quad[\mathrm{pixel}] \\
0.000[\mathrm{~mm}]\end{array}$ \\
\hline $\begin{array}{l}\text { Principal } \\
\text { Point y }\end{array}$ & $\begin{array}{l}1831.480 \\
{[\text { pixel] }} \\
4.396[\mathrm{~mm}]\end{array}$ & $\begin{array}{l}1850.813 \\
{[\text { pixel] }} \\
4.442[\mathrm{~mm}]\end{array}$ & $0.072 \quad[\mathrm{pixel}]$ \\
\hline R1 & -0.263 & -0.267 & 0 \\
\hline R2 & 0.11 & 0.11 & 0 \\
\hline R3 & -0.036 & -0.032 & 0 \\
\hline T1 & 0.001 & 0 & 0 \\
\hline T2 & 0 & 0 & 0 \\
\hline
\end{tabular}

Table 6 shows the relative geographic positioning variance. This figure shows the percentage of relative geographic error between the calibration image and the 
geographic positioning. When the percentage of the image greater than 3 or less than -3 is too high, you need to verify whether you need to adjust the control point accuracy and geographic positioning of the image. It can be seen from the table that $[-3,3]$ is $100 \%$, and the accuracy of the relative geographical position variance of this test meets the requirements.

Table 6. Relative geolocation variance

\begin{tabular}{|l|l|l|l|}
\hline $\begin{array}{l}\text { RelativeGeoloca } \\
\text { tion Error }\end{array}$ & $\begin{array}{l}\text { Images } \\
\mathbf{X}[\%]\end{array}$ & $\begin{array}{l}\text { Images } \\
\mathbf{Y}[\%]\end{array}$ & $\begin{array}{l}\text { Images } \\
\mathbf{Z}[\%]\end{array}$ \\
\hline$[-1.00,1.00]$ & 100.00 & 100.00 & 100.00 \\
\hline$[-2.00,2.00]$ & 100.00 & 100.00 & 100.00 \\
\hline$[-3.00,3.00]$ & 100.00 & 100.00 & 100.00 \\
\hline $\begin{array}{l}\text { MeanofGeolocat } \\
\text { ionAccuracy[m] }\end{array}$ & 5.000000 & 5.000000 & 10.000000 \\
\hline $\begin{array}{l}\text { Sigma of } \\
\text { GeolocationAcc } \\
\text { uracy[m] }\end{array}$ & 0.000000 & 0.000000 & 0.000000 \\
\hline
\end{tabular}

Table 7 shows the absolute geographic positioning variance. The geographical position error is the difference between the initial image and the calculated image, where the minimum error and the maximum error are within 1.5 times. This figure shows that this test meets the accuracy requirements.

Table 7.Absolute geolocation variance

\begin{tabular}{|l|l|l|l|l|}
\hline $\begin{array}{l}\text { Min } \\
\text { Error } \\
{[\mathbf{m}]}\end{array}$ & $\begin{array}{l}\text { MaxE } \\
\text { rror } \\
{[\mathbf{m}]}\end{array}$ & $\begin{array}{l}\text { Geolocati } \\
\text { on Error } \\
\mathbf{X}[\%]\end{array}$ & $\begin{array}{l}\text { Geolocati } \\
\text { on Error } \\
\text { Y[\%] }\end{array}$ & $\begin{array}{l}\text { Geolocati } \\
\text { on Error } \\
\text { Z[\%] }\end{array}$ \\
\hline- & -15.00 & 0.00 & 0.00 & 0.00 \\
\hline-15.00 & -12.00 & 0.00 & 0.00 & 0.00 \\
\hline-12.00 & -9.00 & 0.00 & 0.00 & 0.00 \\
\hline-9.00 & -6.00 & 0.00 & 0.00 & 0.00 \\
\hline-6.00 & -3.00 & 0.00 & 0.00 & 0.00 \\
\hline-3.00 & 0.00 & 54.95 & 52.20 & 53.30 \\
\hline 0.00 & 3.00 & 45.05 & 47.80 & 46.70 \\
\hline 3.00 & 6.00 & 0.00 & 0.00 & 0.00 \\
\hline 6.00 & 9.00 & 0.00 & 0.00 & 0.00 \\
\hline 9.00 & 12.00 & 0.00 & 0.00 & 0.00 \\
\hline 12.00 & 15.00 & 0.00 & 0.00 & 0.00 \\
\hline 15.00 & - & 0.00 & 0.00 & 0.00 \\
\hline Mean[m] & 0.000000 & 0.000000 & 0.000001 \\
\hline Sigma [m] & 0.038905 & 0.044081 & 0.133323 \\
\hline RMSError [m] & 0.038905 & 0.044081 & 0.133323 \\
\hline
\end{tabular}

\section{Conclusions and prospects}

This paper takes Zhanwei New Village in Hongshan District, Wuhan City, Hubei Province as the research object, uses DJI drone to select the snake-shaped flight and aerial photography data, and arranges the corresponding image control points on the ground to complete the image collection. In this experiment, after the data collection was completed, a three-dimensional model was constructed based on PIX4D to generate a point cloud and complete three-space encryption. Then, the occlusion analysis and texture repair were performed on the occlusion part of DSM, and the principle of digital differentiation was corrected based on high-precision DSM Eliminate the relevant parallax, and finally complete the production of real shot images. This experiment has further expanded the application field of drones, providing a quick, simple and low-cost method for the production of real shot images.

\section{References}

1. D.R. Li, M. Wang, J. Pan., F. Hu, Concept, principle and realization of seamless stereoscopic orthography database. Journal of wuhan university (information science edition), 11:950-954.(2007)

2. J.X. Zhang, J.X. Lin, Y.H. Zhang, W.L. Meng, Production of orthography without DEM support. Journal of remote sensing, 3:202-207.(2000)

3. F. Deng, P.L. Li, Y.X. Kan, J.H. Kang, F. Wan, A screening detection method in the production of true projective image of integral projection of digital architectural model. Journal of wuhan university (information science edition), 42(01):97-102.(2017)

4. J.Q. Zhang, B. Xu, M.W. Sun, Y. Zhang, Production of real projective images by triangulation screening. Journal of wuhan university (information science edition), 37(03): 326-329.(2012)

5. D.L. Ma, J. Cui, N. Ding, J.W. Wang, The invention relates to a digital orthophoto image making method. Science of Surveying and Mapping, 38(04):188-189+199. (2013)

6. P. Zhang, Discussion on the technology and problems of making digital orthophoto image. Bulletin of surveying and mapping, 10:28-30.(2003)

7. J.Y. Yang, Y.S. Zhang, Q.F. Bao, X.L. Zou, Research on the detection method of occlusion area made by real projective image. Science of surveying and mapping, 36 (02):37-39.(2011)

8. X.W. Zhang, Research on real project-based image production based on uav image. China university of geosciences (Beijing), (2016)

9. X.M. Zhou, X.L. Meng, X.P. Zhang, Y.H. Mi, Method of constructing the true three-dimensional model of urban tilt photogrammetry. Science of surveying and mapping, 41 (09):159-163.(2016)

10. B.B. Fan, Q.S. Yang, Q. Bao, Z.P. Xu, Production and application evaluation of real projective image of $1: 1000$ in the survey of village cadastral records. Bulletin of surveying and mapping, 10:76-80.(2016)

11. X.L. Feng, K. Wang, L.M. Lou, Production of orthographic image of remote sensing image based on ERDAS. Remote sensing technology and application, 3:176-179.(2003)

12. T. Xia, W.N. Yang, H.H. Liu, J.F. Wang, Extraction of relative DEM and orthophoto map by ASTER stereo image pair. Science of surveying and mapping, 3:144-145+198.(2007) 
13. X.M. Liu, Y.L. Li, F.S. Guo, X.N. Wang, Production of digital orthophotograph based on full digital photogrammetry system. Science of surveying and mapping, 35 (S1):198-199.(2010)

14. M. Meng, J.L. Li, Y.C. Shen, Discussion of Efficient 3D Object Modeling Based on UAV(DJI) and Pix4D. Yunnan geographic environment research, 28(4):1-7, 76.(2016)

15. D.L. Ma, J. Cui, N. Ding, J.W. Wang, A method for making digital orthography. Science of surveying and mapping , 38 (04): 188-189+199.(2013)

16. X.Z. Cai, X.X. Lu, B.C. Long, Discussion on the production method of real projective image based on aerial photography of uav. Surveying and mapping and spatial geographic information, 38 (09):60-62.(2015)

17. A. Lucieer, S. Jong, D. Turner, Mapping Landslide Displacements Using Structure from Motion ( SfM) and Image Correlation of Multi-temporal UAV Photography. Progress in Physical Geography, 38(1):97-116.(2014)

18. M.Y. AI, Q. Hu, J.E. Li, A Robust Photogrammetric Processing Method of Low-altitude UAV Images. Remote sensing, 7(3):2302-2333.(2015)

19. S.F. Liang, Y.L. Wang, L.J. Liu, L.P. San, Production method of orthography based on uav image. Journal of qinghai university (natural science edition), 30(04):54-58.(2012)

20. Z.Q. Zuo, Z.X. Zhang, J.Q. Zhang, H. Cao, Intelligent detection of large scale orthography Mosaic line in xiacheng district assisted by DSM. Journal of surveying and mapping, 40 (01):84-89.(2011)

21. T. Wang, Research on algorithm in real projective image production. Chinese academy of surveying and mapping, (2009)

22. X.R. Xu, Z.X. Li, Method of bridge target recognition in natural landscape. Journal of zhejiang university(natural science edition), $\quad 29$ (3):2752281.(1995) 